\title{
Invasive liver abscess syndrome predisposed by Klebsiella pneumoniae related prostate abscess in a nondiabetic patient: a case report
}

\author{
Chen-Yi Liao ${ }^{1,3}$ (1), Ya-Sung Yang ${ }^{2}$, Yen-Cheng Yeh ${ }^{1}$, Ren-Jy Ben ${ }^{1}$, Ching-Chang Lee ${ }^{1}$, Chi-chang Tsai ${ }^{1}$, \\ Chien-Yao Wang ${ }^{1}$, Wu-Hsien Kuo ${ }^{1}$ and Chih-Chiang Wang ${ }^{1,3^{*}}$
}

\begin{abstract}
Background: Prostate abscess is usually a complication of acute urinary tract infection. Invasive liver abscess syndrome is characterized with Klebsiella pneumoniae related multiple organ metastasis. Concomitant pyogenic liver abscess and prostate abscess have rarely been reported. Recurrent episode of liver abscess is even rarer.

Case presentation: We report a 71-year-old male with acute bacterial prostate abscess and urinary tract infection caused by K. pneumoniae associated with multiple liver abscess, psoas muscle abscess and osteomyelitis. Blood culture and urine culture yielded K. pneumoniae, which confirmed the diagnosis of invasive liver abscess syndrome caused by K. pneumoniae. The patient was successfully treated with empirical antibiotics for 6 weeks.

Conclusions: This case emphasizes the importance of timely and accurate diagnosis followed by appropriate treatment in disseminated $K$. pneumoniae infection to prevent significant morbidity and mortality.
\end{abstract}

Keywords: Invasive liver abscess syndrome, Klebsiella pneumoniae, Prostate abscess

\section{Background}

Prostate abscess is an uncommon but potentially serious disorder with a mortality rate of $6-30 \%$ before the advent of effective antibiotic therapy. Prostate abscess tends to occur in young and middle-aged men. Entry of the microorganisms into the prostate gland almost always occurs via the urethra. Gram-negative infections, especially with Enterobacteriaceae are most common and rarely correlated with Klebsiella pneumoniae infection [1]. Clinical symptoms together with an edematous and tender prostate on physical examination should prompt a diagnosis of acute prostatitis. Although most patients with acute prostatitis respond well to antibiotic therapy, a variety of complications can occur, including bacteremia,

\footnotetext{
*Correspondence: ndmcndmcndmc@gmail.com

${ }^{1}$ Department of Medicine, Kaohsiung Armed Forces General Hospital, No.2, Zhongzheng 1st Road, Lingya District, Kaohsiung 802, Taiwan, ROC Full list of author information is available at the end of the article
}

epididymitis, chronic bacterial prostatitis and prostate abscess. Invasive liver abscess syndrome with concomitant prostate abscess is rare and seldom been reported [2-5]. Herein we present a non diabetic patient with recurrent liver abscess and concomitant prostate abscess predisposed by K. pneumoniae.

\section{Case presentation}

A 71-year-old male with past medical history of liver cirrhosis presented with a 5-day history of general malaise, dysuria and lower abdomen fullness. The patient presented with irritative symptoms such as frequency and nocturia. Obstructive symptoms such as poor urine stream, terminal dribbling and incomplete voiding.

He denied nausea, bowel habit change, body weight loss and fever. He had past liver abscess history with complete resolution 3 years previously. His family history and operation history were unremarkable. On examination, vital signs were stable, and abdominal examination 
showed unremarkable finding and digital rectal examination showed an extremely tender boggy prostate. Investigations showed, white blood cell (WBC) count of $33,000 / \mu \mathrm{L}$ with band form $15 \%$, neutrophil $81 \%$, hemoglobin of $13.9 \mathrm{~g} / \mathrm{dL}$, platelet of $51,000 / \mu \mathrm{L}$, C-reactive protein of $9.62 \%$, blood urea nitrogen of $86 \mathrm{mg} /$ $\mathrm{dL}$, creatinin of $2.1 \mathrm{mg} / \mathrm{dL}$, total bilirubin of $2.48 \mathrm{mg} /$ $\mathrm{dL}$, direct bilirubin of $0.88 \mathrm{mg} / \mathrm{dL}$, albumin of $2.4 \mathrm{~g} / \mathrm{dL}$, AST of $79 \mathrm{U} / \mathrm{L}$, ALT of $64 \mathrm{U} / \mathrm{L}$, alkaline phosphatase of $231 \mathrm{U} / \mathrm{L}$; with PSA total: $15.786 \mathrm{ng} / \mathrm{mL}(0-4)$, PSA free: $0.255 \mathrm{ng} / \mathrm{mL}(<0.934)$, and alpha-feto protein: $1.02 \mathrm{ng} / \mathrm{mL}$ (1.09-8.04). HIV and serology for hepatitis B and C were negative. Urine analysis revealed pyuria with urine white blood cells of too numerous to count; blood cultures and urine culture showed growth of $K$. pneumoniae. Chest radiography and KUB revealed unremarkable findings. Abdominal computed tomography demonstrate multiple lobulated liver abscess with a large measurement of about $3.2 \times 4 \mathrm{~cm}$ without air fluid level. The abscess involved segment IV, segment V, segment VI, segment VII, and segment VIII (Fig. 1). The urinary bladder was thickened secondary to urinary tract infection. The prostate and

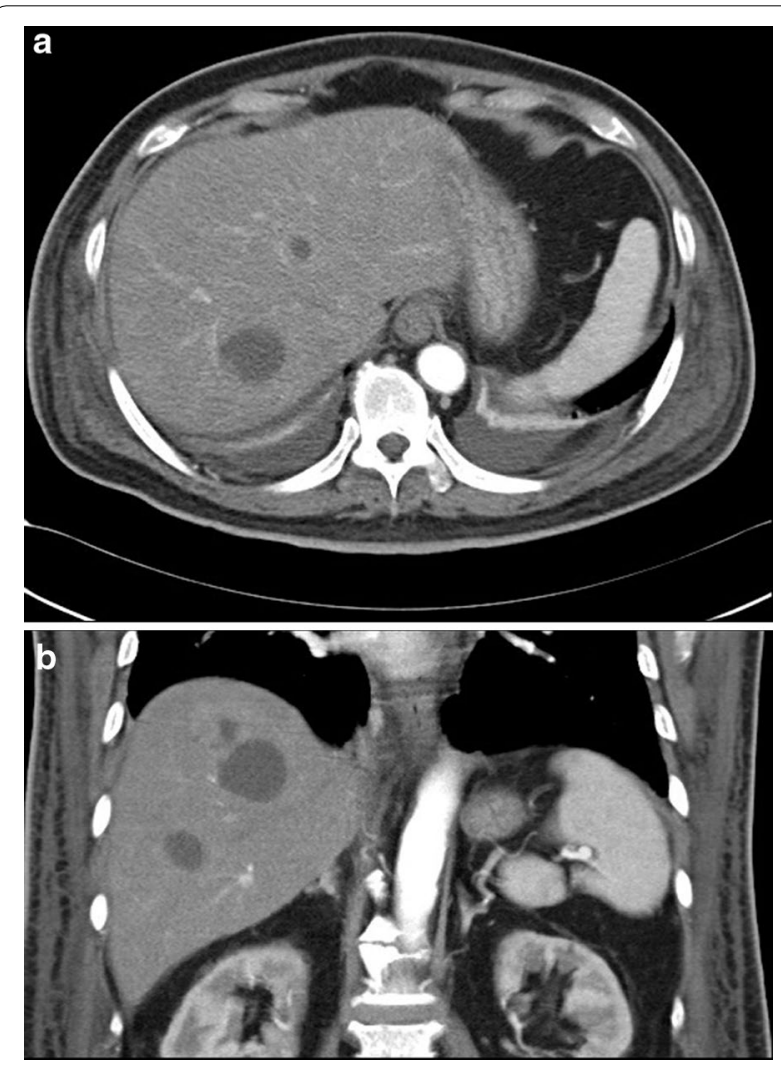

Fig. 1 a, b Contrast-enhanced abdominal CT with contrast denoted numerous lobulated hypodensity lesions in liver compatible with liver abscess overlying segment IV, segment $\mathrm{V}$, segment VI, segment VII, and segment VIII seminal vesicle was enlarged and hypodense, having fluid density compatible with prostate abscess formation with the right one measuring about $4.3 \times 2.4 \mathrm{~cm}$ and left one measured about $4.3 \times 3.3 \mathrm{~cm}$ and seminal vesicle abscess measured about $3.8 \times 3.1 \mathrm{~cm}$. Calcification within the urethral wall was noted (Fig. 2a). No evidence of endophthalmitis could be discerned.

The patient was initially started with cefazolin treatment but progressive low back pain, hydrocele and debilitation developed. Repeat contrast enhanced abdominal CT demonstrated progressive liver abscess, prostate abscess and emerging psoas muscle abscess (Fig. 2b). MRI of pelvis demonstrated osteomyelitis over right pubic symphysis (Fig. 3). Antibiotic was shifted to ceftriaxone $2.0 \mathrm{~g}$ iv QD for better penetration, and the patient's clinical condition gradually improved after 6 weeks of empiric antibiotic treatment. The final capsular serotype of $K$. pneumoniae was $\mathrm{K} 1$ and genotyping revealed rmpA1, rmpA2 (+) and aerobactin $(+)$.
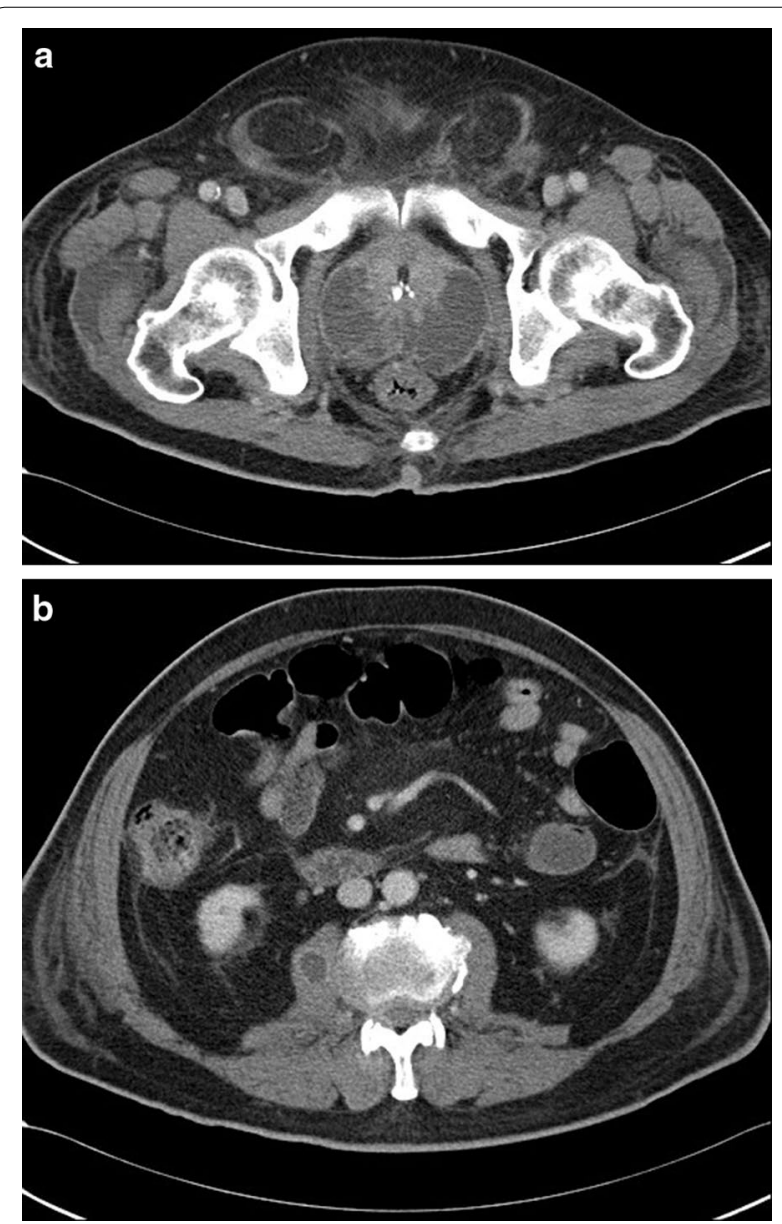

Fig. 2 a Contrast enhanced abdominal CT depicted prostate abscess with calcification noted in the urethral wall. b Contrast enhanced abdominal CT dipicated right psoas muscle abscess 


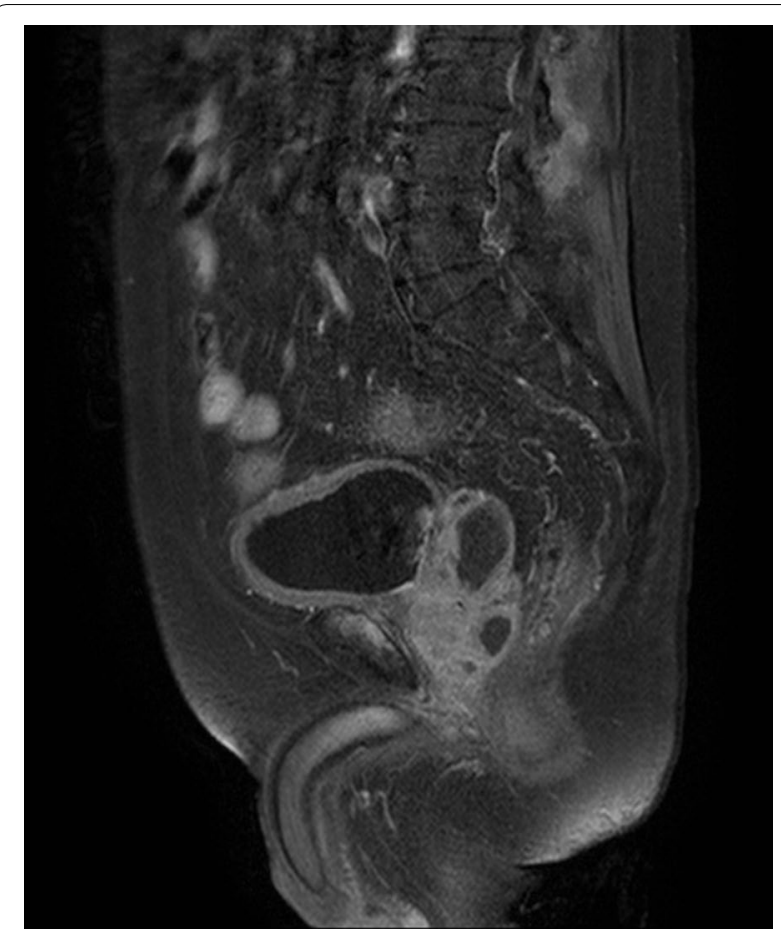

Fig. 3 Fat suppressed MRI of pelvis denoted a hyperintensity lesion (white arrow) over the right pelvis below the bladder wall compatible with osteomyelietis

\section{Discussion}

Invasive liver abscess syndrome is defined by K. pneumonia, which is isolated from the abscess aspirate or blood of a patient with imaging findings consistent with a liver abscess in the absence of underlying hepatobiliary disease [6].

In Taiwan, the serotypes $\mathrm{K} 1$ and $\mathrm{K} 2$ of $\mathrm{K}$. pneumoniae are accompanied with presence of the magA and rmpA genes, which contribute to the virulence of hypermucoviscosity and resistance to in vitro phagocytic uptake by neutrophils as risk factors for metastatic infection [7-10]. Risk factors include patients with impaired host defenses (eg, diabetes mellitus, alcoholism, malignancy, chronic obstructive pulmonary disease, and glucocorticoid therapy). The most common manifestations of metastatic infection are endophthalmitis, meningitis and brain abscess. Other manifestations include lumbar or cervical spondylitis and discitis, septic pulmonary emboli, lung abscess, splenic abscess, necrotizing fasciitis, neck abscess, cerebral abscess, purulent meningitis, otitis media, osteomyelitis, arthritis, prostate abscesses, pylephlebitis and psoas muscle abscesses [11-13].

Diabetes is a frequent underlying illness in reports of $K$. pneumoniae liver abscess, but our patient lacked that putative risk as well. The risk factors in our patient included liver cirrhosis, previous liver abscess and urethral calcification.

Previous liver abscess denote that the liver structure or hepatobiliary system blood flow may be partially destructed which impairs the Kupffer's cells and renders invasive liver abscess syndrome. Six independent risk factors have been proposed predicting severe complications of $K$. pneumoniae liver abscess including: thrombocytopenia $\left(<100,000 / \mathrm{mm}^{3}\right)$, alkaline phosphatase $>300 \mathrm{U} / \mathrm{L}$, gas formation in the abscess, APACHE III score $>40$, use of cefazolin (instead of extended spectrum cephalosporin), and delayed drainage. The initial platelet count in our patient was $51,000 / \mathrm{mm}^{3}$ with alkaline phosphatase of $231 \mathrm{U} / \mathrm{L}$, and use of cefazolin initially along with delayed drainage contributed to progression of the invasive liver abscess syndrome.

Relapse of K. pneumoniae liver abscess after adequate treatment is rare.

The K1 serotype and rmpA gene in our patient might play important roles as predominant virulent factors which leading to phagocytic resistance [14].

The liver has dual blood supply: sterile arterial blood from the hepatic artery and venous blood from the gut where transient bacteremia of the portal system is not unusual. The high relapse rate of $K$. pneumoniae related liver abscess in our case is probably due to the calcified urethra, which rendered the bacteria colonization.

Except for empiric antibiotic treatment, pigtail catheter drainage is the major treatment strategy for liver abscess unless multiple micro-abscess are present, in which case, fine needle aspiration is satisfactory for both diagnosis and treatment. The patient's clinical course is usually uneventful if successful pigtail catheter drainage is combined with a 3-week course of parenteral antimicrobial treatment. Pigtail catheter drainage is usually continued for 1-2 weeks, and the drain is removed when culture of the liver abscess become sterile with daily drainage amount $<5 \mathrm{~mL}$ for several days, and defervescence occurs even after the drainage tube is clamped. Oral antimicrobial treatment for 1-2 months after discharge from the hospital will consolidate the effect of treatment [15]. Our patient declined aggressive intervention and successfully recovered with conservative antibiotic treatment.

\section{Conclusions}

This case emphasizes the importance of timely and accurate diagnosis followed by appropriate treatment in disseminated K. pneumoniae infection to prevent significant morbidity and mortality. In patient with impaired host defenses such as diabetes mellitus or alcoholism related liver cirrhosis, we should always keep invasive liver abscess syndrome in mind. Once this syndrome confirmed, various image study for the metastatic lesion, 
empirical antibiotic use and early adequate drainage of the liver abscess should be undertaken as soon as possible.

\author{
Abbreviations \\ WBC: white blood cell; AST: aspartate aminotransferase; ALT: alanine ami- \\ notransferase; PSA: prostatic specific antigen; HIV: human immunodeficiency \\ virus; KUB: kidney, ureter, bladder; CT: computed tomography; MRI: magnetic \\ resonance imaging.
}

\section{Authors' contributions}

CCW carried out the study and is the original physician of the patient. RJB and YCY participated in infectious survey and interpertate findings of the culture results. YSY contributed to confirmation of the capsular serotype of Klebsiella pneumoniae. WHK participated in providing knowledge of the disease etiology and provide possible differential diagnosis. CYW and CCT collected information of patient and wrote the contents of clinical course of the manuscript. CCL helped draft the manuscript and revised it. CCL and WHK was also in charge of the imaging examinations. All authors read and approved the final manuscript.

\section{Author details}

${ }^{1}$ Department of Medicine, Kaohsiung Armed Forces General Hospital, No.2, Zhongzheng 1st Road, Lingya District, Kaohsiung 802, Taiwan, ROC. ${ }^{2}$ Department of Infection, Tri-Service General Hospital, No.325, Sec. 2, Chenggong Road, Neihu District, Taipei 114, Taiwan, ROC. ${ }^{3}$ Department of Nephrology, Tri-Service General Hospital, No.325, Sec. 2, Chenggong Road, Neihu District, Taipei 114, Taiwan, ROC.

\section{Acknowledgements}

We thank to Mr. Steve (language editor) for involving in drafting the manuscript and revising it critically for important intellectual content. No obvious source(s) of funding noted.

\section{Competing interests}

The authors declare that they have no competing interests.

\section{Consent}

Written informed consent was obtained from the patient for publication of this Case report and any accompanying images.

Received: 15 January 2016 Accepted: 27 July 2016

Published online: 09 August 2016

\section{References}

1. Cornia PB, Takahashi TA, Lipsky BA. The microbiology of bacteriuria in men: a 5-year study at a Veterans' Affairs hospital. Diagn Microbiol Infect Dis. 2006:56:25-30.
2. Chew LC. Septic monoarthritis and osteomyelitis in an elderly man following Klebsiella pneumoniae genitourinary infection: case report. Ann Acad Med Singapore. 2006;35(2):100-3.

3. Kim JY, Ahn KR, Han SW, Baek HR, Lee EJ, Lee CB, Kim ES. Emphysematous prostatitis combined with a liver abscess caused by Klebsiella pneumoniae. Infect Chemother. 2011;43(1):64-7. doi:10.3947/ic.2011.43.1.64.

4. Kim JH, Yang WJ, Kim TH. Klebsiella pneumoniae-induced prostate abscess: how to work it up? Can Urol Assoc J. 2014;8(11-12):E841-4. doi:10.5489/ cuaj.2155.

5. Keller JJ, Tsai MC, Lin CC, Lin YC, Lin HC. Risk of infections subsequent to pyogenic liver abscess: a nationwide population-based study. Clin Microbiol Infect. 2013;19(8):717-22. doi:10.1111/1469-0691.12027.

6. Shin SU, Park CM, Lee Y, Kim EC, Kim SJ, Goo JM. Clinical and radiological features of invasive Klebsiella pneumoniae liver abscess syndrome. Acta Radiol. 2013;54:557.

7. Lin JC, Chang FY, Fung CP, Xu JZ, Cheng HP, Wang JJ, Huang LY, Siu LK. High prevalence of phagocytic-resistant capsular serotypes of Klebsiella pneumoniae in liver abscess. Microbes Infect. 2004;6(13):1191-8.

8. Lin JC, Siu LK, Fung CP, Tsou HH, Wang JJ, Chen CT, Wang SC, Chang FY. Impaired phagocytosis of capsular serotypes K1 or K2 Klebsiella pneumoniae in type 2 diabetes mellitus patients with poor glycemic control. J Clin Endocrinol Metab. 2006:91(8):3084-7.

9. Siu LK, Fung CP, Chang FY, Lee N, Yeh KM, Koh TH, Ip M. Molecular typing and virulence analysis of serotype K1 Klebsiella pneumoniae strains isolated from liver abscess patients and stool samples from noninfectious subjects in Hong Kong, Singapore, and Taiwan. J Clin Microbiol. 2011:49(11):3761-5. doi:10.1128/JCM.00977-11.

10. Ku YH, Chuang YC, Yu WL. Clinical spectrum and molecular characteristics of Klebsiella pneumoniae causing community-acquired extrahepatic abscess. J Microbiol Immunol Infect. 2008;41(4):311-7.

11. Cheng DL, Liu YC, Yen MY, Liu CY, Wang RS. Septic metastatic lesions of pyogenic liver abscess. Their association with Klebsiella pneumoniae bacteremia in diabetic patients. Arch Intern Med. 1991;151(8):1557-9.

12. Chang FY, Chou MY. Comparison of pyogenic liver abscesses caused by Klebsiella pneumoniae and non-K. pneumoniae pathogens. J Formos Med Assoc. 1995;94(5):232-7.

13. Fung CP, Chang FY, Lee SC, Hu BS, Kuo BI, Liu CY, Ho M, Siu LK. A global emerging disease of Klebsiella pneumoniae liver abscess: is serotype $\mathrm{K} 1$ an important factor for complicated endophthalmitis? Gut. 2002:50(3):420-4

14. Cheng HP, Siu LK, Chang FY, Cheng HP, Siu LK, Chang FY. Extendedspectrum cephalosporin compared to cefazolin for treatment of Klebsiella pneumoniae-caused liver abscess. Antimicrob Agents Chemother. 2003:47(7):2088-92

15. Wang JH, Liu YC, Lee SS, Yen MY, Chen YS, Wang JH, Wann SR, Lin HH. Primary liver abscess due to Klebsiella pneumoniae in Taiwan. Clin Infect Dis. 1998;26(6):1434-8.

\section{Submit your next manuscript to BioMed Central and we will help you at every step:}

- We accept pre-submission inquiries

- Our selector tool helps you to find the most relevant journal

- We provide round the clock customer support

- Convenient online submission

- Thorough peer review

- Inclusion in PubMed and all major indexing services

- Maximum visibility for your research

Submit your manuscript at www.biomedcentral.com/submit
Biomed Central 